\title{
Multifocal haemangiomatous malformation: a case report
}

\author{
G . L. M . K I N G S \\ Department of Thoracic Surgery, Bradford Royal Infirmary
}

\begin{abstract}
Kings, G. L. M. (1975). Thorax, 30, 485-488. Multifocal haemangiomatous malformation: a case report. A rare case of haemangiomatous malformation affecting the mediastinum, spleen, kidney, and liver is described. The literature concerning mediastinal haemangiomas is discussed.
\end{abstract}

\section{CASE REPORTS}

A 15-year-old girl was admitted to hospital in 1967 with a history of mild haemoptysis eight weeks previously during an upper respiratory tract infection. On admission the spleen was palpable $5 \mathrm{~cm}$ below the costal margin. There was no abnormal findings in the respiratory, cardiovascular or alimentary systems.

inVESTigations Haemoglobin was $11.8 \mathrm{~g} / \mathrm{dl}$. A blood film, bleeding and clotting times and biochemistry were normal. Bone marrow biopsy showed a hyperplastic marrow. A chest radiograph (Fig. 1) showed a lobulated opacity at the left hilum extending towards the left lower lobe. Bronchoscopy showed the larynx, trachea, and carina to be normal. No abnormality was seen in the bronchial tree.

OPERATION Cystic swellings were found in the upper mediastinum between the vagus and phrenic nerves, in the oblique fissure extending into the upper and lower lobes, and around the hilum of the lung. All of these cysts were found to contain heavily bloodstained fluid.

The spleen, palpable through the diaphragm, was found to contain multiple cysts.

Pathology of lung Biopsies from the cysts of the mediastinum, lower lobe, and oblique fissure were reported as showing lymphangiomatous spaces with papillary walls which were densely fibrous but had traces of smooth muscle. One specimen was intimately mixed with lung showing chronic venous congestion.

There followed an uneventful postoperative period during which an intravenous pyelogram was performed and proved normal. Radio- therapy was given to the mediastinum and left lower lobe during the following six months. A chest radiograph one year later showed no change in the size of the mass and there had been no further haemoptyses.

During subsequent outpatient attendances the size of the spleen was noted to be gradually increasing, and in June 1972 the haemoglobin had dropped to $12.2 \mathrm{~g} / \mathrm{dl}$. A red cell life span study showed this to be reduced from the expected time of 55 days to 23 days, the uptake being principally in the spleen. The patient had developed discomfort in the left hypochondrium and left flank over the enlarged spleen, and splenectomy was thought to be indicated.

OPERATION The spleen was exposed via a left thoraco-abdominal incision and was found to be enlarged and hard, appearing to consist almost entirely of red and purple cysts containing bloodstained fluid (Fig. 2). The liver and left kidney were found to be of normal size but both contained dark coloured cysts, approximately $5 \mathrm{~mm}$ in diameter in the liver and $2 \mathrm{~mm}$ in the kidney. The pancreas was normal.

The spleen was removed and an uneventful postoperative period followed.

Pathology of spleen (Dr. H. S. Kellett) The spleen was greatly enlarged, weighing $1200 \mathrm{~g}$, but of normal shape. The surface was grey with white spots visible through the capsule. The cut surface showed almost contiguous cysts, from 1 to $10 \mathrm{~mm}$ in diameter, containing milky-white inspissated fluid.

Histological sections showed most of the spleen to be replaced by an angioma of mainly cavernous structure. Most of the vascular spaces had weakly 

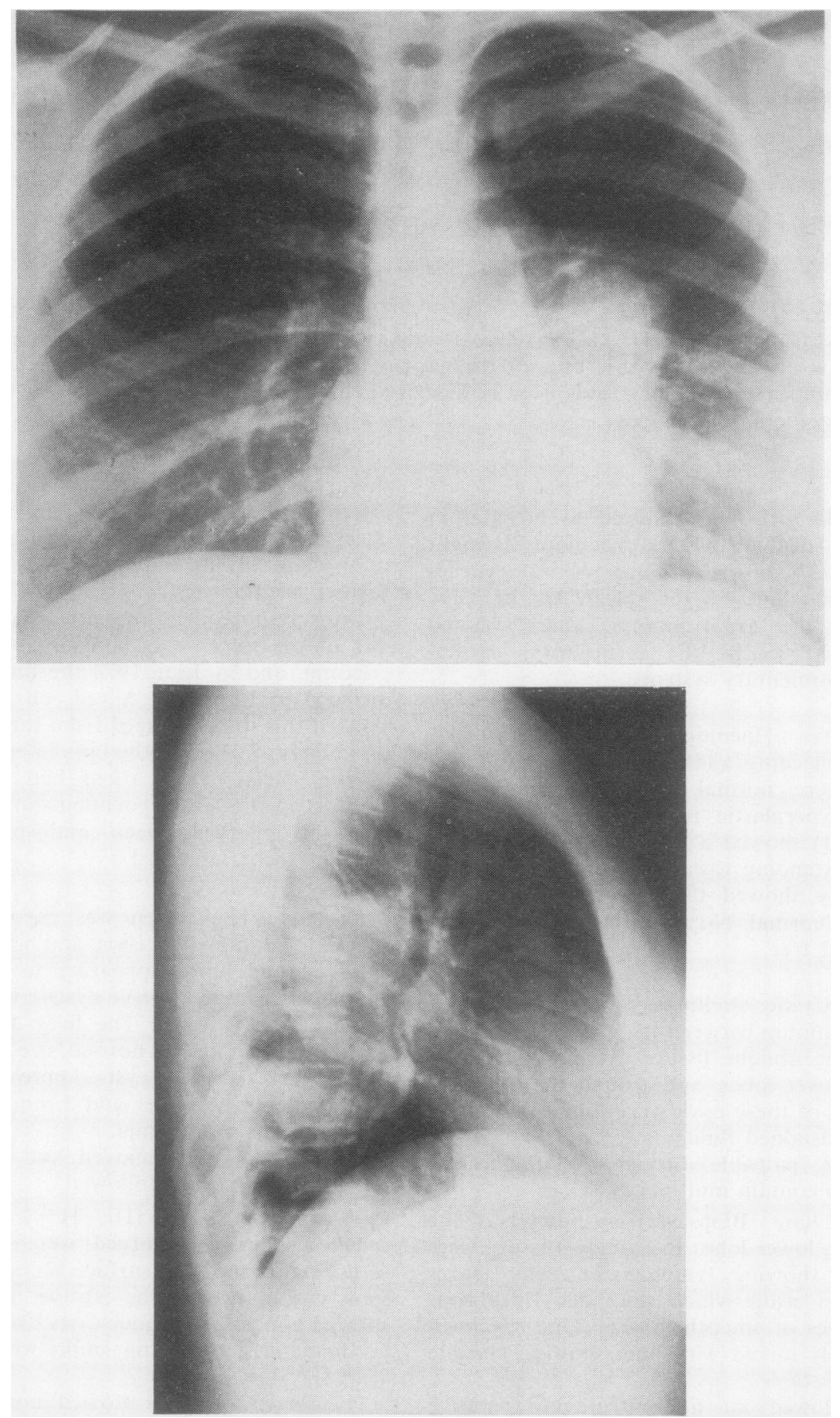

FIG. 1. Postero-anterior and right lateral radiographs showing lobulated lesion at right hilum. 


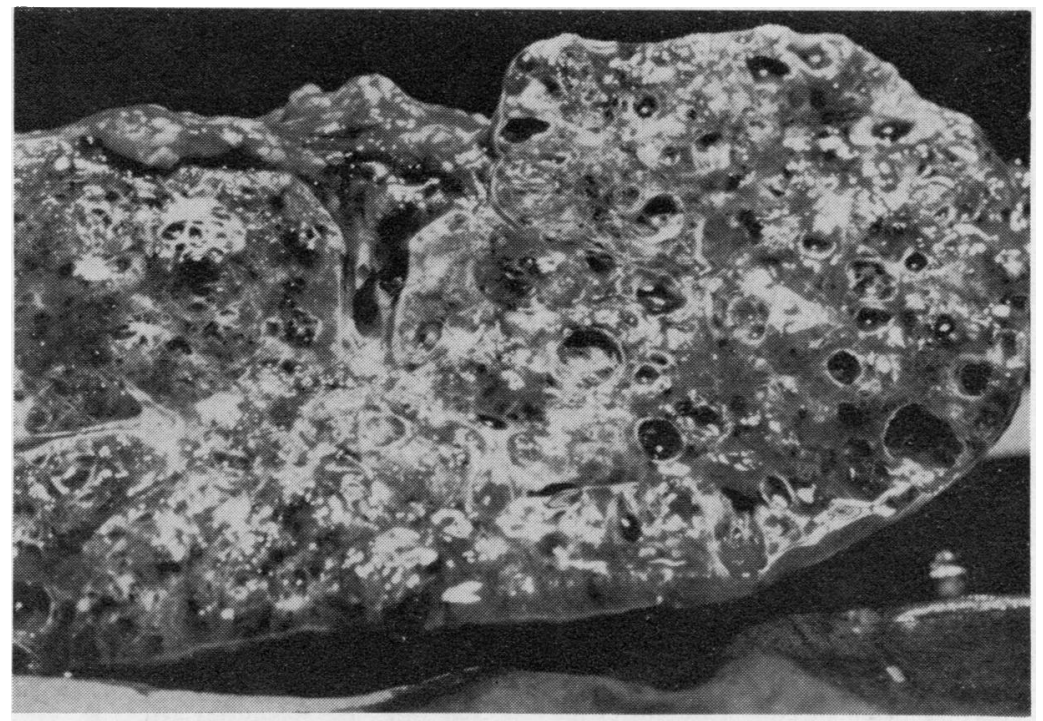

FIG. 2. Longitudinal section of spleen removed at surgery showing multiple cysts.

eosinophilic contents, but some contained a number of red cells and others were filled with blood. Many of these cystic vessels were separated by varying amounts of stroma, recognizable in occasional fields as normal splenic pulp along with small lymphocytic foci.

It was difficult to decide whether the condition was a lymphangioma with haemorrhage into some cysts, or a mixed lymphangioma and haemangioma.

The pathology slides were forwarded for the opinion of Professor R. A. Willis who considered that the lesions were more likely to be haemangiomatous because of the regularity of the vascular spaces and their lack of intercommunication.

In view of the operative findings, liver and renal function were assessed and found to be normal. Neither retinoscopy nor brain scanning revealed any abnormality.

The patient returned to work six weeks after operation and has remained asymptomatic.

\section{DISCUSSION}

The haemangioma described in this article is rare in that it involves many viscera, thus failing to comply with any named syndrome of haemangiomatous malformation (Willis, 1967). The slow progress of the disease differentiates this case from those described by Holden and Alexander (1970) in infants and young children.
Mediastinal haemangioma occurs rarely. Attar and Cowley (1964), in a review of the literature, found that they accounted for only $1.5-3 \%$ of mediastinal tumours, and Baker (1967) found only 70 cases reported in the world literature. These lesions may occur at almost any age but are commonest in infancy and childhood. The clinical presentation is variable and, apart from those discovered at routine chest radiography, Larson et al. (1961) found that the modes of presentation, in decreasing order of frequency, were cough, expectoration, dyspnoea, and haemoptysis. Radiography shows a lobulated mediastinal mass which may contain calcification. Treatment consists of surgical excision where possible. Attar and Cowley (1964), reviewing 27 cases treated by complete excision, found no evidence of recurrence up to eight years after operation. In those cases where only partial excision was possible there was usually postoperative improvement, and radiotherapy was not found to be effective since only $20 \%$ of tumours showed evidence of regression.

Multifocal haemangiomata simultaneously affecting several tissues or organs are infrequently encountered in the literature but certain combinations are recognized and are described by Willis (1967). In all but one of these named syndromes there are associated haemangiomata of the skin, in contrast to the findings in this patient. The remaining syndrome is known as 
Lindau's disease, first described in 1926, and reviewed by Melmon and Rosen (1964). The distribution of the visceral involvement is similar to that in our patient but there are also retinal and cerebral haemangiomata present in the full syndrome. Melmon and Rosen describe examples of the delayed appearance of retinal and cerebral changes but the length of observation of the case described here makes this diagnosis unlikely. Parsons and Ebbs (1940) reported two cases of multifocal haemangiomata with involvement of the thoracic and abdominal viscera similar to that of our case. Both patients were young women in whom no cerebral or retinal lesions were found, but in both cases the long bones were also affected.

The difficulty encountered in this case with regard to the histology of the spleen emphasizes the similarity in appearance of haemangioma and lymphangioma. This is further complicated by the fact that both lesions may occur simultaneously (Stanley, 1966). These features are probably explained by the embryology of the two tissues, the lymph vessels arising as outgrowths of cells from parent veins (Childress, Baker, and Samson, 1956), the subsequent development of both lymphatic and vascular channels being closely related. The principal histological features of these two lesions have been well described by Childress et al (1956), Goldberg (1940), Pachter and Lattes (1963), and Harrow (1957).

I should like to thank Professor R. A. Willis for the pathological report, and the Medical Photographic Department of St. Luke's Hospital, Bradford.

\section{REFERENCES}

Attar, S. and Adams Cowley, R. (1964). Haemangioma of the mediastinum. American Surgeon, 30, $141 . \overline{0}$

Baker, J. M. (1967). Unusual vascular tumors of the

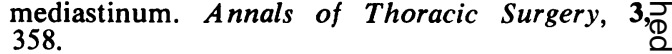

Childress, M. E., Baker, C. P.. and Samson, P. C.œ (1956). Lymphangioma of the mediastinum. Journal of Thoracic Surgery, 31, 338.

Goldberg, S. A. (1940). Primary splenic neoplasms. $\overrightarrow{\vec{\omega}}$ American Journal of Clinical Pathology, 10, $700 \mathrm{\omega}$

Harrow, B. R. (1957). Retroperitoneal lymphatic cyst. Journal of Urology, 77, 82.

Holden, K. R. and Alexander, F. (1970). Diffuse neonatal haemangiomatosis. Paediatrics, 46, 411.

Larson, D. L., Myhre, B. A., Schmidt, E. R., andô Jaeschke, W. H. (1961). Lymphangioma in unusual sites, spleen, mesentery, retroperitoneum, mediastinum and the greater omentum.Wisconsin Medical Journal, 60, 279.

Melmon, K. L. and Rosen, S. W. (1964). Lindau'so disease. American Journal of Medicine, 36, 595.

Pachter, M. R. and Lattes, R. (1963). Mesenchymal tumors of the mediastinum. Cancer, 16, 95.

Parsons, L. G. and Ebbs, J. H. (1940). Generalized angiomatosis presenting the clinical characteristics of storage reticulosis. Archives of Disease? in Childhood, 15, 129.

Stanley, K. E. (1966). Haemangioma-lymphangioma of the bladder in a child. Journal of Urology, 96, 51 .

Willis, R. A. (1967). Pathology of Tumours, 4th $\overrightarrow{\bar{\sigma}}$ edition, p. 727. Butterworth, London.

Requests for reprints to: G. L. M. Kings, FRCS Department of Thoracic Surgery, Bradford Royalo Infirmary, Bradford. 